\title{
An Environment For Domestic Supervised Amblyopia Treatment
}

\author{
Giancarlo Facoetti, Angelo Gargantini, and Andrea Vitali \\ Department of Engineering, University of Bergamo (BG), Dalmine, Italy \\ \{giancarlo.facoetti, angelo.gargantini, andrea.vitali1\}@unibg.it, \\ WWW project page: http://3d4amb.unibg.it
}

\begin{abstract}
Amblyopia (also called lazy eye) is a condition in which the eye and the brain do not work properly together; this condition causes poor vision in the lazy eye. It involves around $4 \%$ of the children. We have devised a system for the diagnosis and treatment of amblyopia by using $3 D$ technology. To be successful, the proposed treatment must be enjoyable and suitable for domestic use (for instance by watching TV) and carried out with a constant supervision by the doctors. We present a system in which patients and doctors exchange information about the prescribed activities.
\end{abstract}

Amblyopia (also called lazy eye) is a condition in which the eye and the brain do not work properly together; this condition causes poor vision in the lazy eye. It is a neurological process, as the problem is caused by poor transmission of the visual stimulus through the optic nerve. If amblyopia is not diagnosed and treated in the first years of life, the lazy eye becomes weaker and the normal eye becomes dominant. The traditional way to treat amblyopia is carried out wearing a patch over the normal eye for several hours a day, through a treatment period of several months. This treatment has some drawbacks: it is unpopular, not well accepted by the young patients, and sometimes can disrupt the residual fusion between the eyes.

Our group has been involved in the use of computer technologies for disabilities [3] for several years. The project 3D4AmB exploits the stereoscopic 3D technology, that through glasses with active shutters permits to show different images to the amblyotic eye and the normal eye. We developed some software both for amblyopia diagnosis and treatment that uses this kind of 3D technology. Since the patients are young children, we decided to implement the diagnosis and treatment modules in a form of videogame, in order to make the treatment fun and not boring. The final aim of the project is to give the patients a complete domestic computer based treatment that can be used at home. In fact, a common personal computer mounting a commercial 3D board and a 3D monitor or $\mathrm{TV}$ is enough to run the software developed for the 3D4AmB project.

In this work we present a complete environment for domestic and supervised amplyopia treatment. At this stage the environment is under development and validation in collaboration with medical personnel. The treatment begins with the evaluation of the patient in presence of the doctors. Once the amblyopia level 
is evaluated and patient case file is created, the treatment stage can be carried out at home. We have developed several software modules in order to emulate the two main types of treatment which are defined as passive and active treatments. The difference between them is based on the interaction of patients during sessions. The passive treatment consists of a video player with some filters applied to the normal eye, such as blurring and luminosity and contrast decreasing. The patient, wearing the $3 \mathrm{D}$ glasses, can watch movies and cartoons, and the software will apply the filters to the normal eye in a gradual way and following the parameters set by the doctors in the patient case file. This treatment emulates the classical patching, but it allows partial and dynamic occlusion.

The active module consists in a videogame platform. This choice is based on clinical results which demonstrated the increase of brain skills (in particular visual-spatial skills) on children with visual disorders after some videogame sessions. The games include a modified tetris, a space invaders, and an open source Mario Bros clone to which we have added the stereoscopic 3D vision. In the stereoscopic version, every character of the game can be shown either to the normal eye or to the weak eye (binocular vision). It is also possible to show a character to an eye for a given percentage, and to the other for the remaining value (for example, $70 \%$ to the weak eye and $30 \%$ to the normal eye). This allows to differently exercise the two eyes (as shown in the image above). As always, how to show the game characters and the left/right eyes percentages has been initially set by the doctors in the patient case file.

During the home treatment, our software records some parameters like the time spent viewing the clips and movies (passive treatment) and playing videogames (active treatment). Periodically the user is tested for stereoacuity at home and this measure is used to judge a possible improvement of the sight. Such data are sent to the doctors through the Internet periodically so they can follow patient progress and intervene if necessary. The doctor can set the treatment parameters in the patient case files according to these results. Moreover our software can autonomously decide to change (under certain bounds) the parameters of the treatment and to suggest some types of activity in order to improve efficiency.

Section 1 introduces amblyopia, the classical treatments and our 3D-based proposal. We present in Section 2 the extension of our environment to a distributed scenario in which patients and doctors exchange data about the treatments by Internet. The architecture of the system is presented in Section 3.

\section{Using 3D for amblyopia treatment and diagnosis}

Amblyopia, otherwise known as 'lazy eye', is reduced visual acuity that results in poor or indistinct vision in an eye that is otherwise physically normal, or out of proportion to associated structural abnormalities. Typically amblyopia is present in only one eye and is generally associated with a squint or unequal lenses in the prescription spectacles. This low vision is not correctable (or only partially) by glasses or contact lenses. 
There exist several causes of amblyopia. Anything that interferes with clear vision in either eye during the critical period (birth to 6 years of age) can result in amblyopia. The most common causes of amblyopia are constant strabismus (constant turn of one eye), anisometropia (different vision/prescriptions in each eye), and/or blockage of an eye due to trauma, lid droop, etc. If one eye sees clearly and the other sees a blur, the good eye and brain will inhibit the eye with the blur. The brain, for some reason, does not fully acknowledge the images seen by the amblyopic or lazy eye. Thus, amblyopia is a neurologically active process. The inhibition process (suppression) can result in a permanent decrease of the vision in that eye that can not be corrected with glasses, lenses, or surgery. This condition affects $2-3 \%$ of the population, which equates to conservatively around 10 million people under the age of 8 years worldwide. Children who are not successfully treated when still young (generally before the age of 7) will become amblyopic adults. As amblyopic adults, they will have a normal life, except that they are prohibited from some occupations and they are exposed to a higher risk of losing the good eye due to injury or eye disease and became seriously visually impaired.

Amblyopia is currently treated by wearing an adhesive patch over the nonamblyopic eye for several hours per day, over a period of several months. This treatment was introduced in the 18th century and is commonly used also nowadays. This conventional patching or occlusion treatment for amblyopia often gives disappointing results for several reasons: it is unpopular, prolonged, and it can sometimes make the squint worse because it disrupts whatever fusion there is [6]. These issues frequently results in poor or non-compliance and since the success of patching depends on compliance, it performs on average very poorly. The treatment by itself works well, but it is often abandoned because it is too much trouble to take. Very often, children are averse to wearing a patch and parents found occlusion difficult to implement [4]. As noted in [11], a treatment whose unacceptability is greater than the motivation of the patients to apply it, will be often abandoned. And if the treatment of patching is not continued, it will eventually fail [10]. For this reason, the orthoptists and ophthalmologists are continuously looking for a more acceptable solution to the problem, i.e. an effective treatment that is also complied with and so really works [9].

There exist several attempts to introduce variations to occlusion which could perform nearly as well as the occlusion without the problem of compliance and the risks of disruption of any existing binocularity. In particular, partial occlusion consists in wearing an adhesive filter to attenuate the vision of the good eye [2]. This method is also known as penalization and it can be also performed optically by using defocusing lens or pharmaceutically by using atropine which causes blurring of the sound eye. In [1], the authors experimented the occlusion of the lens over the preferred eye with a translucent tape. This technique permitted uninterrupted and prolonged occlusion, with a successful visual outcome. However, this kind of treatment has still some problems of compliance and applicability (e.g. pharmaceutical penalization must be administered by a physician). There are some attempts to use computer systems to implement a sort 
of virtual penalization, called rebalancing. In [5], the authors introduce a vision system based on a head mounted display (HMD) which performs rebalancing of the vision by using a simultaneous enhancing/attenuation image adjustment. The image presented to the normal eye is attenuated while the image presented to the amblyopic eye is enhanced. The main problem of such system is that binocular HMDs have a limited wearability for young children, they either have a very low resolution and a limited weight or a good resolution but a considerable weight, they are costly, and not easily extensible.

The main goal of our research project has been devising a system for vision rebalancing that is accessible. With the term "accessible" we mean: inexpensive (with a low cost), friendly to use, suitable for domestic use, enjoyable, and easily extensible.

All the characteristics listed above should reduce the compliance problem and make the proposed treatment acceptable. In [7] we have presented a system, called 3D4AMB, which is based on 3D vision technologies and which is extended in this paper in a complete environment for domestic supervised amblyopia treatment. The devised system is based on the 3D technologies, although its goal is not to provide the patients with the 3D experience but to allow binocular vision. The classical use of a 3D system is to provide the two eyes with two different images of the same scene with a slightly offset viewing angles which correspond to the different viewpoints of our left and right eye. This vision produces an illusion of real depth of the scene and it is the basis of the $3 D$ virtual reality. We exploit only the capability of the 3D system to send two different images to the eyes while we do not want to recreate a virtual reality.

The working prototype of the proposed system we have already built, is based on the NVIDIA@ 3 D Vision ${ }^{\text {TM }}$ technology, although other 3D technologies may be supported as well in the future. The NVIDIA 3D Vision technology is one of the most accessible 3D technologies available on the market today, it requires a standard personal computer with a NVIDIA graphic card (also entry level NVIDIA graphic boards work), a monitor 3D Vision ready, which is capable of a refresh rate of $120 \mathrm{Mhz}$, and a NVIDA 3D glasses. The NVIDIA 3D vision is based on LCD active shutter technology. With this technology, the left and right eye images are presented on alternating frames, but since the monitors used are capable of $120 \mathrm{~Hz}$, each eye still sees a full $60 \mathrm{~Hz}$ signal that is equivalent to the refresh rate on LCD monitors today. This offers a number of advantages with respect to other stereoscopic technologies like polarized or anaglyphs glasses or head-mounted displays, including: full image quality per eye, wide viewing angle for $3 \mathrm{D}$, and acceptable cost. The system we have developed for 3D4AmB consists in a normal PC desktop connected to a 3D monitor (3D Vision-Ready Display). The PC must be 3D capable and have all the 3D4AMB software installed on it. The patient wears the NVIDIA active LCD shutter glasses that allow viewing a different image from the left and right eye. The scenario is depicted in Figure 1 and explained below.

Diagnosis The first use of 3D4AmB software is for the detection and measurement of amblyopia. We have developed some specific diagnosis software 


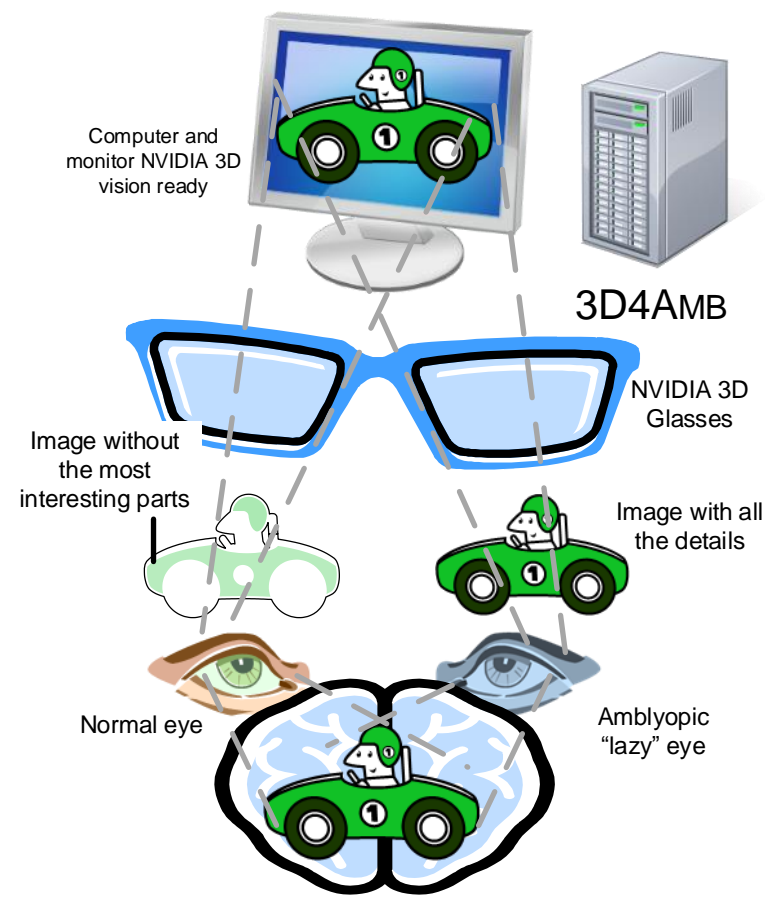

Fig. 1. Video rebalancing: basic principle

that replicates and improves the traditional tests for amblyopia diagnosis and stereoacuity measurement [13]. The tests are presented through the software in a form of videogame and can highlight the presence of amblyopia. Furthermore, the tests give as output the level of weakness of the lazy eye, using a contrastbased measurement of suppression. The contrast balance point can be computed by exploiting the dot stimulus concept. The amblyopic patient watches visual stimulus presented dichoptically (separated images for each eye). Stimuli and noise-stimuli are represented with visible points on the screen, grouped in two sets, named signals and noise. Signals move along horizontal direction and noise moves in random directions. The patient has to guess in what direction the signals are moving. The patient interaction should be minimal, he/she only should say which the right direction is. The difficulty is defined as the number of signals shown to the user when he/she chooses the correct direction. The difficulty is modified through variation of signals number, but the system keeps the number of stimuli constant.

Vision rebalancing by Video In [8] we have presented a vision rebalancing system for videos. The basic principle of the system is that the amblyopic or 'lazy' and the normal eye can be shown two different images or videos. This principle can 
be used in practice for video rebalancing, where the amblyopic eye is shown an enhanced version of a video, while the non-amblyopic or 'good' eye is shown a penalized version of the same video (as shown in Fig. 1). The video to be shown by the patient is duplicated by $3 \mathrm{D} 4 \mathrm{AMB}$ in two versions and each version is then modified: one for the right eye (the amblyopic eye in the Figure) is enhanced and one for the left eye (the good eye in the Figure) is penalized. The 3D4AmB software decides how to process the video depending on the type of the desired treatment. In this way, the lazy eye of the child is more stimulated to work, but the non-amblyopic eye is not patched. The patient brain joins (or fuses) the two video versions in one unique vision experience. To make sure that the patient can join the two videos, the two versions must be not too much different. Note that the final video is a bidimensional video because the goal is not to stimulate the stereo vision of the patient (at least initially). We plan to work on the use of real $3 \mathrm{D}$ stereo video streaming to combine vision rebalancing with depth perception.

Videogames We created a rehabilitation environment in a form of video games. To this end, we exploited an open source clone of the well known game Mario Bros, adapting it to our needs. In particular, in the rendering module of the game, we added stereoscopic vision functionalities. In this way, we can select video signal quality independently for the two eyes. (As previously said) The underlying idea is to degrade only the image shown to the normal eye, in order to stimulate the weak eye. To provide the game with an univocal reference scale, we replicated through software the effects of the common occlusion filters. On the user interface it's possible to set the occlusion rate among the following values: $0.0,0.1,0.2,0.3,0.4,0.6,0.8,1.0$, like one would do with the traditional occlusion patches. It's also possible to allocate images on the both two eyes with different percentages. For example, we can send an image with $80 \%$ opacity to the weak eye, and the remaining $20 \%$ to the normal eye. In this way fusion between the two eyes is stimulated.

We provided the rehabilitation game with two different way to operate: in the first option, we apply the software occlusion filters to the whole image shown to the weak eye. In the second options, we apply filters only to some characters and not to the whole image. For examples, we can occlude partially just the main character, or some enemies, leaving the background untouched. A similar work has been presented also in [14].

\section{Use Cases for a Distributed Environment}

In this paper we present an extension of the 3D4AMB environment for the diagnosis and treatment of ambliopya in a geographically distributed setting. The basic assumption is that the patient can perform his/her exercises without the need of an actual visit to the hospital or clinic by exploiting our software which is suitable for domestic use. However, she/he is constantly followed by a physician which can supervise (supported by an automatic system) the patient activities, change prescription and recall the patient for a visit if needed. 
First of all we have homogenize all the different types of activities presented in the previous section. Every activity has the following attributes:

- eye to be penalized, left or right (the normal eye),

- target intensity (from 0.1 to 1 with the analogous of the Bangerter foils),

- temporal kind of application, which can be: instantaneous (the intensity is applied from the beginning of the exercise at prescribed value), periodic (the intensity is applied periodically over the duration of the activity), or ramp (the intensity in gradually increased).

We have devised the following scenario, represented in Fig. 2.

1. A patient goes to a physician (an ophthalmologist or an orthoptist).

2. The physician performs the necessary medical tests (possibly by using also the 3D4AmB software) and saves the patient data on a server. The tests give as output the level of weakness of the lazy eye, to be stored in the patient case file. The doctor can add some information such as treatment time lasting and the level of exercise to be performed by the lazy eye. She/he prescribes a series of activities, each with its attributes (type, target intensity, and application kind).

3. The patient periodically starts the (daily) activity at home with his/her computer to which the 3D4AmB software is installed. At the beginning the $\mathrm{PC}$ connects with the sever and updates the activities prescriptions, if necessary. Depending on the prescriptions and on the activities already done by the patient, the software suggests to start a certain kind of exercise with suitable parameters (intensity and temporal application). The user is still free to choose what to do, but the PC desktop logs every user choice. At the end (or whenever it is possible) the software client sends information to the server.

4. Periodically the physician logs into the server and checks how the patient is performing. If needed, the doctor can adjust the treatment and recall the patient.

\section{Distributed architecture}

The system structure has been designed by following principals guidelines of Health Information Tehcnology (HIT). HIT is the application of information processing involving both computer hardware and software that deals with the storage, retrieval, sharing, and use of health care information, data, and knowledge for communication and decision making [12]. In the last decade, many healthcare software have been developed among which a small set under Free license, such as Health GNU (http://health.gnu.org), FreeMED (http:// freemedsoftware.org) and OpenEMR (http://www.open-emr.org). The most important feature of this system is the distributed web design that permits to manage health care information according to the final user (e.g., patient, physician and healthcare personnel) and his/her credentials. 


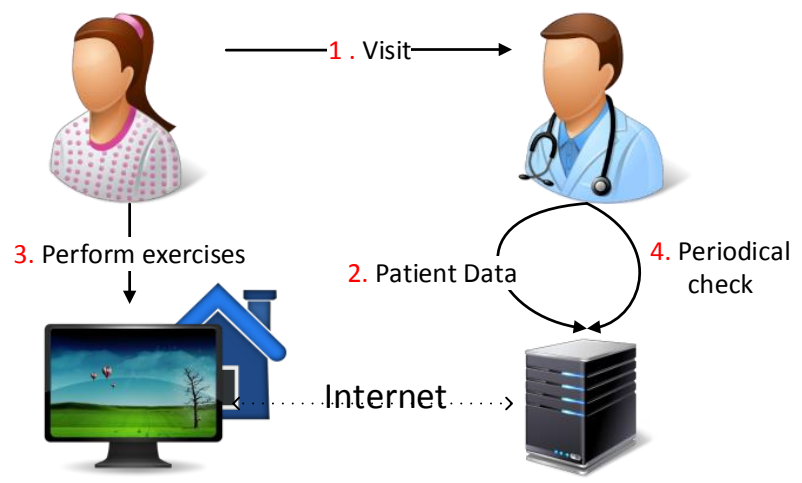

Fig. 2. A typical scenario for domestic supervised treatment

Our system uses the same approach to only manage treatments of visual diseases. The architecture is based on Model-View-Controller pattern for web applications (Fig. 3).

The Model is defined by different modules which permit to manage both patient and treatment data. The information are divided in different modules:

- Patient data. They are composed by both personal information (i.e., first and last name, sex, age) and visual pathologies. In particular, visual pathologies describe the eye condition in terms of visual acuity and improvement level during the rehabilitation. These information are accessible from each actor that uses the distributed platform.

- Diagnostic tests. The doctor associates a set of visual tests in order to make improvements of eye skills. This module contains many parameters, for example the type of diagnostic test, the average duration of each session and medical parameters. The last ones are used from physician to make an evaluation of patient.

- Rehabilitation data. It is a set of parameters which permit to describe the patient's performance during each session of treatment (e.g., duration, level of difficult, number of executed sections).

The modules are managed using database created on server side and it is queried exploiting MySQL DBMS.

The Controller part manages data exchange between database and final user. In particular, the interchange format is defined using XML language which permits to describe information in a consistent way. In order to introduce a standardization of health information, we chosen to use ICD-10 to standardize the description of visual pathologies. For example, instead of typing an arbitrary 


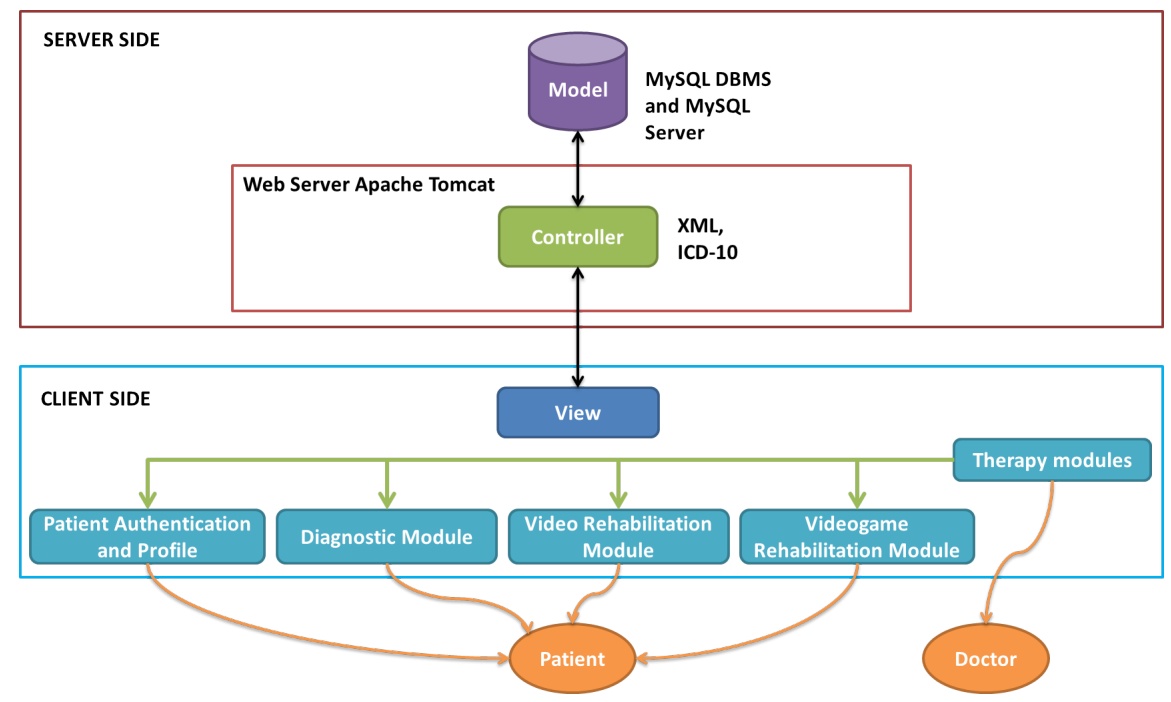

Fig. 3. 3D4AmB system architecture

name for the condition, the doctor will be able to choose from over $14000 \mathrm{mu}-$ tually exclusive classified diseases (http://www.who.int/classifications/icd/en/). In particular, some visual diseases are classified as showed in table 1.

\begin{tabular}{|l|l|}
\hline ICD-10 Code & Description \\
\hline H53.009 & Amblyopia, unspecified, unspecified eye. \\
\hline H53.039 & Strabismic amblyopia, unspecified eye. \\
\hline H53.010 & Subjective visual disturbances, unspecified. \\
\hline H53.139 & Sudden visual loss, unspecified eye. \\
\hline H53.2 & Diplopia. \\
\hline H53.30 & Disorder of binocular vision, unspecified. \\
\hline H53.34 & Suppression of binocular vision. \\
\hline H53.33 & Simultaneous visual perception without fusion. \\
\hline
\end{tabular}

Table 1. Some example of ICD-10 codes about visual disease

The View is defined from both web pages and software for diagnosis and therapy. Furthermore, a data sharing policy has been defined to distinguish information which are only accessible from physicians or generic health personnel. This choice has been made because the patients may wrongly interpret some medical parameters without a medical explanation.

This part defines the client side of 3D4AMB that is formed by applications used from either patient or doctors. A patient can access to his/her health profile through the use of an authentication module released from hospital. The patient profile shows a summary of each treatments which the patient is undergoing. The 
other patient's modules (i.e., diagnostic module, video rehabilitation module and videogame rehabilitation module) are downlodable applications which permit to execute the prescribed therapy.

A physician can use patient data by exploiting the therapy module. This module is a set of web pages which permit the doctor to manage therapy information for his/her patients. In particular, he/she prescribes activities by modifying some parameters of treatments modules. Furthermore, therapy module allows the doctor an evaluation of therapies. Therefore, doctor delivers the prognosis that will be showed to the patient through the web page of patient profile.

\section{Conclusions}

We have presented in this paper an environment for providing the treatment for amblyopia in a geographically distributed setting. The proposed system allows a more continuous control by the physicians of the actual progress of the patients, which on the other side, can perform the activities at home without the need of frequent visits to the hospital. We are validating the proposed environment with the collaboration of the local hospital. We are also evaluating the threats to the validity of the current approach. One is that there is no real control over the identity of the patients and (more seriously) about the actual use of the system. For instance, the patient could not wear the glasses or not actually watch the movies as prescribed. We are studying some counter-measures about these problems (for instance by checking periodically during the activities if the user is actually using the system as prescribed).

\section{References}

1. R. G. Beneish, R. C. Polomeno, M. E. Flanders, and R. K. Koenekoop. Optimal compliance for amblyopia therapy: occlusion with a translucent tape on the lens. Can J Ophthalmol, 44(5):523-528, Oct 2009.

2. W. N. Charman. Optical characteristics of transpaseal as a partial occluder. Am J Optom Physiol Opt, 60(10):846-850, Oct 1983.

3. G. Colombo, G. Facoetti, and C. Rizzi. A digital patient for computer-aided prosthesis design. Interface Focus, 3(2):82-84, Feb. 2013. 00001.

4. M. Dixon-Woods, M. Awan, and I. Gottlob. Why is compliance with occlusion therapy for amblyopia so hard? a qualitative study. Arch Dis Child, 91(6):491494, June 2006.

5. S. Fateh and C. Speeg. Rebalancing the visual system of people with amblyopia "lazy eye" by using HMD and image enhancement. In R. Shumaker, editor, VMR '09 Proceedings of the 3rd International Conference on Virtual and Mixed Reality: Held as Part of HCI International 2009, volume 5622 of Lecture Notes in Computer Science, pages 560-565. Springer, 2009.

6. S. L. Fawcett. Disruption and reacquisition of binocular vision in childhood and in adulthood. Curr Opin Ophthalmol, 16(5):298-302, Oct 2005.

7. A. Gargantini. Using $3 \mathrm{D}$ vision for the diagnosis and treatment of amblyopia in young children. In International Conference on Health Informatics HEALTHINF $2011,2011$. 
8. A. Gargantini, M. Bana, and F. Fabiani. Using 3D for rebalancing the visual system of amblyopic children. In Virtual Rehabilitation (ICVR), 2011 International Conference on, pages $1-7$, june 2011.

9. R. Gregson. Why are we so bad at treating amblyopia? Eye, 16(4):461-462, July 2002.

10. D. Newsham. Parental non-concordance with occlusion therapy. British Journal of Ophthalmology, 84(9):957-962, 2000.

11. A. Searle, P. Norman, R. Harrad, and K. Vedhara. Psychosocial and clinical determinants of compliance with occlusion therapy for amblyopic children. Eye, 16(2):150-155, March 2002.

12. T. G. Thompson and D. J. Brailer. The decade of health information technology: delivering consumer-centric and information-rich health care. Washington, DC: US Department of Health and Human Services, 2004.

13. A. Vitali, G. Facoetti, and A. Gargantini. An environment for contrast-based treatment of amblyopia using 3D technology. In International Conference on Virtual Rehabilitation 2013 - August 26-29, 2013 in Philadelphia, PA, U.S.A., 2013.

14. H. Wei, Y. Zhao, F. Dong, G. Saleh, X. Ye, and G. Clapworthy. A cross-platform approach to the treatment of ambylopia. In Bioinformatics and Bioengineering (BIBE), 2013 IEEE 13th International Conference on, pages 1-4, Nov 2013. 